\title{
CAPITULO 88 \\ RISCO DE LESÃO POR PRESSÃO PRECOCE EM PACIENTES COM INCONTINÊNCIA URINÁRIA E FECAL NA UNIDADE DE TERAPIA INTENSIVA: REVISÃO INTEGRATIVA
}

DOI 10.4322/978-65-995353-2-1.c88

\begin{abstract}
$\underline{\text { Antonia Mylene Sousa Almeida }}^{{ }^{1}}$, Isabella Cristina Galvão da Silva ${ }^{\mathbf{2}}$, Maria Samila Silva Carvalho Andrade ${ }^{3}$, Danilo da Silva Soares ${ }^{4}$, Ludmilla Rafaela Marinho da Silva ${ }^{5}$, Pamella Souza Macedo ${ }^{6}$, Victor Emmanuel Costa Ribeiro ${ }^{7}$, Francisco Lucas Leandro de Sousa $^{8}$, Daniele Teixeira dos Santos ${ }^{9}$, Brenda Kelly da Silva Monte ${ }^{10}$

${ }^{1}$ Faculdade de Educação São Francisco (mylenesousa123@hotmail.com)

${ }^{2}$ Faculdade de Educação São Francisco, (icgds1234@gmail.com)

${ }^{3}$ Centro Universitário do Piauí, (samilacarvalhoccb@gmail.com)

${ }^{4}$ Centro Universitário Maurício de Nassau, (enferdanilo.alves@ gmail.com)

${ }^{5}$ Pontifícia Universidade Católica de Minas Gerais, (ludmillarafaela30@gmail.com)

${ }^{6}$ Escola Superior de Ciências da Santa Casa de Misericórdia de Vitória, (pamellamacedo20@gmail.com)

${ }^{7}$ Associação de Ensino Superior do Piauí, (emmannuelribeiro36@ gmail.com)

${ }^{8}$ Centro Universitário Maurício de Nassau, (lucasleandro2912@gmail.com)

${ }^{9}$ Uninassau Unidade Dorotéias, (danieleteixeira02@hotmail.com)

${ }^{10}$ Mestre em Enfermagem pela Universidade Federal do Piauí, (bksm@faesf.com.br)
\end{abstract}

\section{Resumo}

O objetivo desse estudo é analisar o risco do desenvolvimento precoce de lesão por pressão em pacientes com incontinência urinária e fecal em unidade de terapia intensiva. Trata-se de uma revisão integrativa da literatura, no qual O levantamento dos artigos se deu através das bases de dados Medical Literature Analysis and Retrieval System Online (MEDLINE via BVS), Literatura Latino-americana e do Caribe em Ciências da Saúde (LILACS via BVS) e Base de Dados de Enfermagem (BDENF via BVS). Para esse trabalho, foram considerados como critérios de inclusão os artigos originais disponíveis por meio eletrônico em português e/ou em inglês. Como critério de exclusão, foi adotado artigos que não tratam da temática proposta, artigos duplicados, teses, monografias, dissertações, artigos de revisão, livros e que não apresenta o texto completo. O método de busca foi realizado através dos descritores em ciências da saúde (DeCS): "Lesão por pressão", "Incontinência urinária" ; "Incontinência fecal" e "Unidade de terapia intensiva". A pergunta norteadora para essa pesquisa foi a seguinte: Qual risco de desenvolver precocemente lesão por pressão em pacientes que sofrem de incontinência

\section{E - book Tripé do Ensino Superior: Ensino,} Pesquisa e Extensão 
urinária e fecal em uma unidade de terapia intensiva? Dentre os principais resultados, a higiene do paciente é o cuidado mais básico em que os profissionais de enfermagem devem realizar e priorizar. A incontinência urinária e fecal é vista como fatores para o desenvolvimento de lesão por pressão precocemente, pois a umidade e mobilidade são categorias que influenciam e limitam o paciente por não conseguir deambular sem auxilio ou não deambular totalmente. Contudo, implementar medidas para evitar danos sérios à pele merece atenção no âmbito da saúde e segurança do paciente. Conclui-se então, que o risco de desenvolver a LP precocemente devido a incontinência uriária e fecal na unidade de terapia intensiva é evidente na lituratura.

Palavras-chave: Lesão por pressão; Incontinência urinária; Incontinência fecal; Unidade de terapia intensiva

Área Temática: Ciências da Saúde

E-mail do autor principal: mylenesousa123@ hotmail.com

\section{INTRODUÇÃO}

A lesão por pressão (LP) é definida por um prejuízo situado na pele e/ou tecidos moles subjacentes sobre uma proeminência óssea ou relacionada ao uso de dispositivo médico ou artefato (MENDONÇA et al., 2018). A LP é um sério problema de saúde pública e tem sido fonte de preocupação, pois leva ao desenvolvimento de transtornos físicos, emocionais e influencia na morbimortalidade (SOARES; HEIDEMANN, 2018).

Estudos apontam que o risco para o desenvolvimento da lesão por pressão no Brasil é entre $41,2 \%$ e $59 \%$ e há uma prevalência entre $8 \%$ e $23 \%$. Essas estimativas são um fator preocupante, tendo em vista que é um evento que pode ser prevenido em até $95 \%$ dos casos de acordo com a declaração do Rio de Janeiro sobre a prevenção de lesão por pressão (SOARES; HEIDEMANN, 2018).

A lesão por pressão é dividida em quatro estágios. O estágio 1, a pele encontra-se íntegra com eritema e pode aparecer diferente em pele com pigmentação escura. $\mathrm{O}$ estágio 2, ocorre a perda de espessura parcial da pele com exposição da derme. Já a lesão por pressão estágio 3, ocorre a perda total da espessura da pele na qual o tecido adiposo é visível. E o último, estágio 4 que ocorre a perda total da espessura da pele com exposição ou palpação direta de tecidos como fáscia, músculo, tendão, ligamento, cartilagem ou osso, podendo ser visíveis (SANTOS et al., 2019).

Os locais mais frequentes para desenvolver a LP é na região sacra, trocânteres, calcâneos e proeminências ósseas. Há vários fatores para o desenvolvimento dessa lesão, no qual o principal é a compressão exercida sobre um capilar, entre uma estrutura óssea e uma superfície. Os aspectos que predispõe sua ocorrência são a pressão, o atrito, desequilíbrio nutricional, idade 
avançada, diabetes, medicamentos e a umidade, que compreende a incontinência fecal e urinária (SILVA et al., 2020).

Segundo Silva et al. (2020), a incontinência urinária e fecal é definida como uma condição que ocorre perda involuntária de urina e fezes, aumenta o risco de desenvolver a lesão por pressão devido a umidade, o contato com a pele, dentre outros fatores. Em vista disso, na unidade de terapia intensiva (UTI) é o lugar na qual os pacientes se encontram vulneráveis e existem fatores que prejudicam sua estrutura psicológica, além do surgimento de complicações como a lesão por pressão.

Contudo, essa pesquisa se torna relevante devido a importância de se entender os principais fatores que levam ao desenvolvimento da lesão por pressão em pacientes com incontiência urinária e fecal, além de estudar e verificar o risco associado a esse problema. A pergunta que norteou essa pesquisa foi qual risco de desenvolver precocemente lesão por pressão em pacientes que sofrem de incontinência urinária e fecal em uma unidade de terapia intensiva? $\mathrm{O}$ presente trabalho tem como objetivo analisar o risco do desenvolvimento precoce de lesão por pressão em pacientes com incontinência urinária e fecal em unidade de terapia intensiva.

\section{MÉTODO}

Trata-se de uma revisão integrativa da literatura, na qual tem como propósito produzir resultados alcançados em pesquisas sobre uma temática/questão de forma organizada, sistemática e integral. Esse método permite a inclusão de pesquisas experimentais e não experimentais, bem como dados da literatura teórica e empírica, possibilitando uma compreensão mais completa do tema (ERCOLE; MELO; ALCOFORADO, 2014).

De acordo com Souza, Silva e Carvalho (2010), a revisão integrativa é um instrumento da prática baseada em evidências, bem como um tipo de método que auxilia na produção de informações e na aplicação dos resultados obtidos. Para construir essa revisão, é necessário de seis etapas, sendo elas: a criação da pergunta norteadora; busca de dados; coleta de dados; análise dos conteúdos selecionados; discussão dos resultados; apresentação da revisão (ERCOLE; MELO; ALCOFORADO, 2014).

A pergunta norteadora para essa pesquisa foi a seguinte: Qual risco de desenvolver precocemente lesão por pressão em pacientes que sofrem de incontinência urinária e fecal em uma unidade de terapia intensiva?

O levantamento dos artigos se deu através das bases de dados Medical Literature Analysis and Retrieval System Online (MEDLINE via BVS), Literatura Latino-americana e do Caribe em Ciências da Saúde (LILACS via BVS) e Base de Dados de Enfermagem (BDENF via BVS). Para esse trabalho, foram considerados como critérios de inclusão os artigos originais

\section{E - book Tripé do Ensino Superior: Ensino, Pesquisa e Extensão}


disponíveis por meio eletrônico em português e/ou em inglês. Como critério de exclusão, foi adotado artigos que não tratam da temática proposta, artigos duplicados, teses, monografias, dissertações, artigos de revisão, livros e que não apresenta o texto completo.

O método de busca foi realizado através dos descritores em ciências da saúde (DeCS): "Lesão por pressão", "Incontinência urinária"; "Incontinência fecal" e "Unidade de terapia intensiva", como também através do Medical Subject Headings (MeSH): "Pressure Ulcer”, "Urinary Incontinence", "Fecal Incontinence" and "Intensive Care Units", com recorte temporal nos últimos 10 anos (2011 a 2021), anos correspondentes ao maior número de pesquisas. Foram cruzados através do operador booleano "AND” para busca simultânea dos assuntos.

\section{RESULTADOS E DISCUSSÃO}

Através do levantamento nas bases de dados, foram encontrado um total de 159 artigos nas bases de dados. Após a filtragem dos artigos, 98 foram eliminados por não disponibilizarem do texo completo, 03 por não estar em português e/ou inglês e 08 por não corresponder aos anos selecionados para o estudo. A partir disso, 50 artigos foram selecionados para a leitura e análise crítica. Após a leitura e análise dos artigos, 02 foram eliminados por serem jornais, 01 por ser livro, 01 por ser relatório de estágio, 23 por não estar de acordo com a temática proposta, 03 foram eliminados por estarem duplicados e 03 por serem artigos de revisão. Diante disso, 17 artigos foram incluídos na pesquisa (Figura 1). 
Figura 1. Levantamento dos artigos nas bases de dados, Pedreiras, Maranhão, 2021.

\begin{tabular}{|c|c|c|}
$\underset{(\mathrm{n}=138)}{\operatorname{MaDLINE}}$ & \begin{tabular}{|} 
LILACS \\
$(\mathrm{n}=12)$
\end{tabular} & $\begin{array}{c}\text { BDENF } \\
(\mathrm{n}=09)\end{array}$ \\
\hline
\end{tabular}
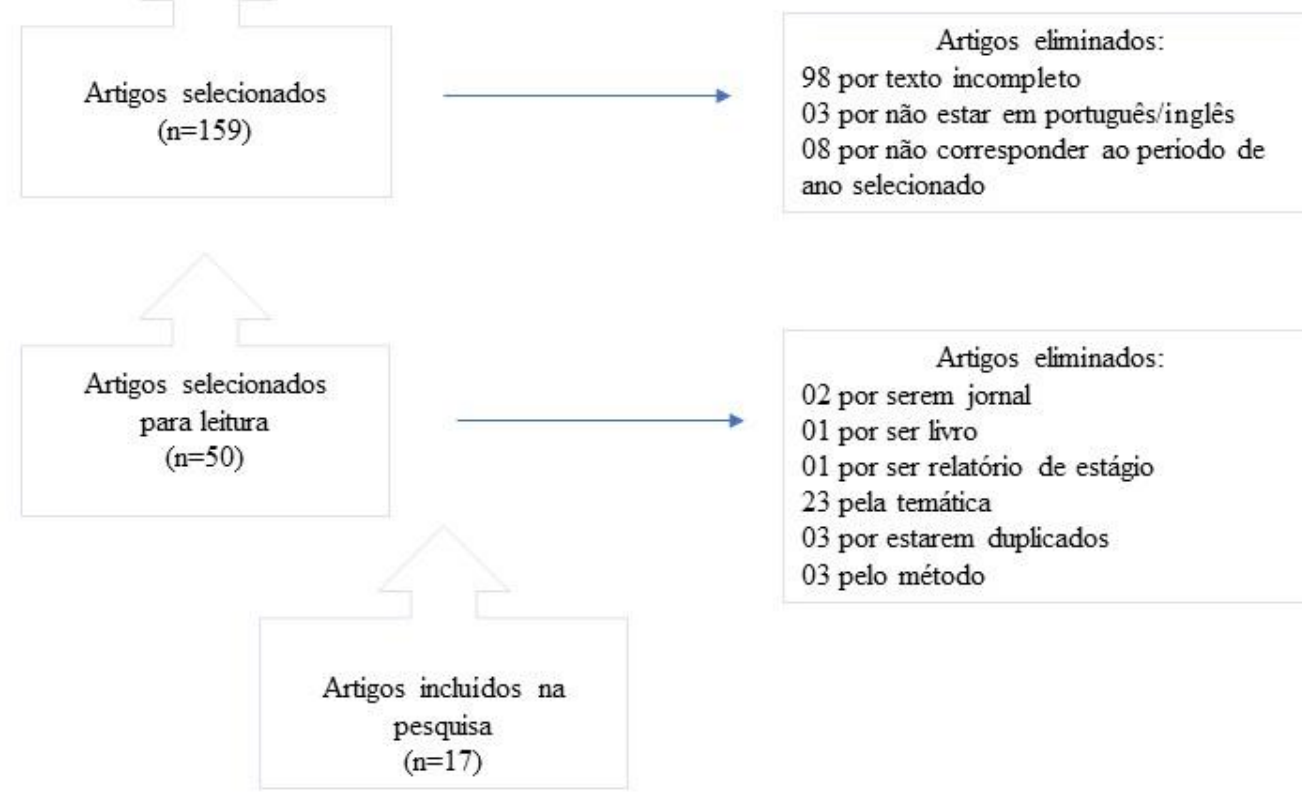

Fonte: Autores, 2021.

Dentre os principais resultados, a higiene do paciente é o cuidado mais básico em que os profissionais de enfermagem devem realizar e priorizar. Dentro da unidade de terapia intensiva, esses cuidados devem se redobrar, pois há o risco de ruptura da pele em pacientes que apresentam incontinência urinária e fecal. Além disso, o liquido ou semi-líquido pode contaminar o ambiente no qual o paciente se encontra e aumentar o risco de infecção e contaminação cruzada (JOHANSEN et al., 2020).

A incontinência urinária e fecal é vista como fatores para o desenvolvimento de lesão por pressão precocemente, pois a umidade e mobilidade são categorias que influenciam e limitam o paciente por não conseguir deambular sem auxilio ou não deambular totalmente. Esses tipos de incontinência são avaliados como fatores de risco com maior impacto sobre lesões graves de pressão (KAYSER et al., 2018).

A umidade e danos superficiais à pele aumentam o coeficiente de fricção e diminui a tolerância a cargas mecânicas. Com isso, um paciente com LP que apresenta incontinência

\section{E - book Tripé do Ensino Superior: Ensino,} Pesquisa e Extensão 
urinária tem a probabilidade de aumentar do estágio I para o estágio IV em relação a pacientes sem incontinência (LACHENBRUCH et al., 2016).

O risco de desenvolver LP em pacientes com incontinência é maior em comparação a pacientes sem incontinência. Em vista disso, os enfermeiros devem fornecer medidas de proteção para esses pacientes que possuem esse problema urinário e fecal e produtos devem ser usados, quando necessários (ZHOU et al., 2018).

Aos pacientes com incontinência, o uso de dispositivos como foley cateteres, ostomias e sistemas de manejo fecal, são essenciais para prevenir a lesão por pressão precoce nas unidades de terapia intensiva. Ademais, esses pacientes que tem esse problema são propensos a desenvolverem a LP devido a umidade do ambiente, fator esse que leva a evolução dos estágios da lesão por pressão (KAYSER, et al., 2018).

Novas diretrizes clínicas para a prevenção de LP incluem manter a pele limpa e seca, como também o desenvolvimento de um plano de gestão para pacientes com incontinência e sua limpeza imediata após a incontinência (GREY; GIULIANO, 2017).

Estudos apontam a relevância do cuidado com a pele através de um pano pré-umedecido, hidratante à base de emoliente e a pele à base de dimeticona para diminuir a prevalência de lesão por pressão associado a incontinência urinária e fecal (GREY; GIULIANO, 2017).

Um dos papeis da enfermagem na UTI é atuar no leito na parte de higienização e mobilização do paciente. Esses cuidados, quando centrados no paciente promovem a segurança do indivíduo diminuindo as prevalências de lesões. Além desses cuidados, outras atividades são necessárias para a prevenção da LP como mudar o paciente de posição, utilizar travesseiro entre as pernas para evitar fricção entre os joelhos e tornozelos, dentre outros (AVSAR; KARADAG, 2017).

Profissionais de enfermagem especializados em feridas, ostomia e inconitência são importantes em um mundo no qual a necessidade é crescente para os cuidados com os pacientes que sofrem com esses problemas. Esses enfermeiros especialistas atuam na gestão de incontinências, além do cuidado com a pele relacionado as incontinências e os dados a pele referente a umidade (BERKE et al., 2019).

\section{CONCLUSÃO}

Conclui-se então, que o risco de desenvolver a LP precocemente devido a incontinência uriária e fecal na unidade de terapia intensiva é evidente na lituratura. Ademais, a lesão por pressão, apesar de ser prevenível, é uma situação recorrente e que variados fatores podem auxiliar para o seu desenvolvimento.

E - book Tripé do Ensino Superior: Ensino, Pesquisa e Extensão 
Além disso, pessoas com incontinência uriánia e fecal encontram-se em constante risco de pele, ferida e complicações relacionadas, e a partir disso, é necessário profissionais especializados com conhecimentos e habilidades para a avaliação do risco, prevenção e tratamento.

Contudo, implementar medidas para evitar danos sérios à pele merece atenção no âmbito da saúde e segurança do paciente. Além de prevenir LP baseado em evidências através de intervenções para redistribuir a pressão como giro regular e reposicionamento do indivíduo, como também cuidados relacionados a pele para promover limpeza, proteção e melhora da pele através de barreiras de umidade.

\section{REFERÊNCIAS}

AVSAR, P; KARADAG, A. Efficacy and Cost-Effectiveness Analysis of Evidence-Based Nursing Interventions to Maintain Tissue Integrity to Prevent Pressure Ulcers and IncontinenceAssociated Dermatitis. Worldviews on Evidence-Based Nursing, v. 00, n. 0, p. 1-8, 2017.

BERKE, C. et al. Papel da Enfermeira de Feridas, Ostomia e Continência em Continence Care: atualização de 2018. J Wound Ostomy Continence Nurs., v. 46, n. 3, p. 221-225, 2019.

ERCOLE, F. F; MELO, L. S; ALCOFORADO, C. L. G. C. Revisão Integrativa versus

Revisão Sistemática. Rev Min Enferm. v. 18. n. 1, p. 1-260, 2014.

GREY, M; GIULIANO, K. K. Dermatite e imobilidade associadas à incontinência como fatores de risco de lesão por pressão. J Wound Ostomy Continence Nurs., v. 00, n. 0, p. 1-5, 2017.

JOHANSEN, E. et al. Moisture associated skin damage (MASD) in intensive care patients: A Norwegian point-prevalence study. Intensive \& Critical Care Nursing, v. 60, e:102889, 2020.

KAYSER, S. A. et al. Predictors of Superficial and Severe Hospital-acquired Pressure Injuries: A Cross-Sectional Study. Revista Internacional de Estudos de Enfermagem, v. 89, p. 46-52, 2019.

LACHENBRUCH, C. et al. Analysis of Incontinence and Hospital-Acquired Pressure Ulcers From the International Pressure Ulcer PrevalenceTM Survey. J Wound Ostomy Continence Nurs. v. 43, n. 3, p. 235-241, 2016. 
MENDONÇA, P. K. et al. Prevenção de lesão por pressão: ações prescritas por enfermeiros de centros de terapia intensiva. Texto Contexto Enferm, v. 27, n. 4:e4610017, 2018.

SANTOS, M. L. et al. Cicatrização de lesão por pressão: abordagem multiprofissional. Rev enferm UFPE on line., v. 13:e239634, 2019.

SOUZA, M. T.; SILVA, M. D.; CARVALHO, R. Revisão integrativa: o que fazer. Einstein, v.8, n. 1, p. 102-6, 2010.

SOARES, C. F; HEIDEMANN, I. T. S. B. Promoção da saúde e prevenção da lesão por pressão: expectativas do enfermeiro da atenção primária. Texto Contexto Enferm, v. 27, n. 2:e1630016, 2018 .

SILVA, J. F. et al. Lesões precoces por pressão em pacientes com incontinência miccional e fecal em unidade de terapia intensiva. REAEnf/EJNC, v. 2:e2591, 2020.

ZHOU, Q. et al. The prevalence and specific characteristics of hospitalised pressure ulcer patients: A multicentre cross-sectional study. 2018. 